\title{
La sécheresse et le droit de l'eau
}

\author{
Anne Bourlet \\ Secrétariat d'Etat à l'environnement
}

\section{Introduction}

Bien que l'eau soit relativement abondante dans un pays tempéré comme la France, elle fait l'objet de nombreux conflits d'usage (alimentation en eau potable, préservation des milieux naturels aquatiques et de la vie piscicole, irrigation, énergie hydraulique). En période de pénurie occasionnée par une sécheresse, ces conflits se trouvent aggravés. En France, il est reconnu que l'un des usages de l'eau apparaît prioritaire : c'est l'alimentation en eau potable.

Il existe dans le droit français très peu de dispositions législatives et réglementaires qui prennent en compte de façon spécifique cette situation relativement exceptionnelle qu'est la sécheresse.

La lutte contre les effets de la sécheresse suppose en réalité de mettre en œuvre l'ensemble des mesures de police et de gestion des eaux.

Après avoir examiné la mise en œuvre de ces mesures générales, on abordera le recours à des dispositions législatives exceptionnelles.

\section{Mesures générales de police et de gestion des eaux}

L'autorité administrative s'est vue conférer dès la Révolution des pouvoirs de police lui permettant de prescrire les mesures nécessaires pour assurer le libre cours des eaux, préserver la salubrité publique, et répartir les eaux au nom de l'intérêt général.

Elle doit les mettre en œuvre pour prévenir et lutter contre la sécheresse.

\subsection{La police des eaux ou la gestion quantitative}

Ces pouvoirs de police des eaux trouvent essentiellement leur fondement dans les dispositions du titre 3 chapitre 2 du code rural et dans celles du titre 3 du code du domaine public fluvial. Ils ne concernent donc essentiellement que les eaux des cours d'eau non domaniaux, et des lacs et des cours d'eau domaniaux, définis par l'article $1^{\text {er }}$ du code du domaine public fluvial et de la navigation intérieure, qu'ils soient inscrits ou rayés de la nomenclature des voies navigables (quelques chiffres en annexe).

Le lit des cours d'eaux non domaniaux appartient aux propriétaires riverains (article 98 du code rural). Ceux-ci ne disposent que d'un simple droit d'usage (article $97 \mathrm{du}$ code rural et 644 du code civil) sur ces eaux qui font partie des choses communes (article 714 du code civil).

Par contre, le lit des cours d'eau et lacs domaniaux, ainsi que le droit d'usage sur leurs eaux appartiennent à l'Etat.

Les pouvoirs de police des eaux ne s'appliquent donc pas aux eaux susceptibles d'appropriation privée telles que les eaux pluviales, les eaux souterraines, les eaux de source, les mares et autres eaux stagnantes. Un contrôle administratif de l'utilisation des eaux souterraines a cependant été introduit par le décret loi du 8 août 1935 et ses décrets d'extension, et par les articles 40 et 57 de la loi du 16 décembre 1964 relative au régime et à la répartition des eaux et à la lutte contre leur pollution, afin d'éviter qu'une utilisation intensive des eaux souterraines ne conduise à l'épuisement des nappes.

Au titre de leurs pouvoirs de police, les préfets réglementent les ouvrages et prises d'eau établis dans le lit des

\section{Drought and water law}

Water is the subject of numerous user conflicts (drinking water supplies, satisfaction of domestic needs, preservation of natural aquatic landscapes, and fish-breeding, irrigation, electricity production using water). In times of shortages these conflicts are seriously aggravated. We will firstly go through the principles of water law, both as regards the legal situation governing water use as well as the water policing powers of the authorities. Then we present the legislative and regulatory provisions which enable a drought to be managed in a very precise way. These provisions are moreover very few and their main aim is to give the authorities the powers to satisfy a usage they judge to be a priority: namely supplies of drinking water. 
cours d'eau (article 106 du code rural et 25 du code du domaine public fluvial). Compte tenu de la rédaction de l'article $107-1^{\circ}$ du code rural, une enquête n'est nécessaire que dans la mesure où l'ouvrage est susceptible d'avoir une influence sur le régime ou le mode d'écoulement des eaux. La condition posée par l'article $107-1^{\circ}$ du code rural a d'ailleurs été reprise dans l'article 4 du modèle de règlement d'eau de 1906.

Le juge a très vite admis que les préfets pouvaient en particulier fixer le débit que le permissionnaire doit maintenir dans le lit du cours d'eau pour satisfaire aux différentes utilisations de l'eau, notamment pour l'alimentation en eau potable (cf. CE 5 août 1906 Ollagnier), et la satisfaction des besoins domestiques (CE 13 juillet 1917 Lefaivre).

L'article 410 du code rural, introduit par la loi du 29 juin 1984, relative à la pêche en eau douce et à la gestion des ressources piscicoles, a fixé par ailleurs un débit minimum " légal " destiné à garantir en permanence la vie, la circulation et la reproduction des espèces qui peuplent les eaux au moment de l'installation de l'ouvrage. Sauf dispositions transitoires, ce débit doit être supérieur ou égal au dixième du module du cours d'eau, si celui-ci est inférieur à $80 \mathrm{~m}^{3} / \mathrm{s}$.

Dans la circulaire adressée aux préfets le 8 septembre 1988, le secrétaire d'Etat chargé de l'environnement a insisté sur la nécessité d'assortir les autorisations qu'ils délivrent, en application tant de l'article 106 du code rural et 25 du code du domaine public fluvial que de l'article 16 du 16 octobre 1919 relative à l'utilisation de l'énergie hydraulique, de prescriptions qui prennent en compte de façon satisfaisante les variations saisonnières du débit du cours d'eau, des autres besoins et des impacts sur le milieu naturel.

Ces autorisations peuvent toujours être supprimées ou modifiées, au titre de la police des eaux, notamment pour répondre aux besoins de l'alimentation en eau potable (article $109-2^{\circ}$ du code rural, article 26 du code du domaine public fluvial).

La fixation du débit réservé pour les ouvrages qui font l'objet d'une déclaration d'utilité publique ou qui relèvent du régime de la concession en application de la loi du 16 octobre 1919 revêt d'autant plus d'importance que les dispositions des articles 109 du code rural et 26 du code du domaine public fluvial ne leur sont pas applicables.

\subsection{La police des rejets ou la gestion qualitative des eaux} Les préfets réglementent par ailleurs, en application de la loi du 16 décembre 1964 relative au régime et à la répartition des eaux et à la lutte contre leur pollution, les rejets susceptibles d'altérer la qualité des eaux, qu'il s'agisse d'eaux superficielles ou souterraines.

La lutte contre la pollution des eaux présente une acuité particulière en période de sécheresse : en effet la pollution chronique de certains cours d'eau risque alors d'atteindre un niveau intolérable en raison de la dilution insuffisante des effluents par les débits naturels et de l'augmentation de la température de l'eau.

Il appartient donc aux préfets de veiller à une application attentive des réglementations diverses qui concourent à la protection des eaux superficielles et souterraines, en tenant compte des caractéristiques hydrobiologiques particulières des régions exposées à la sécheresse :

- autorisations ou déclaration des installations classées pour la protection de l'environnement ;

- création de périmètre de protection des eaux d'alimentation ;

- règlement sanitaire départemental ;

- réglementation spécifique relative aux déchets, aux produits chimiques, aux exploitations de carrière.

Pendant les périodes d'étiage sévère, il convient d'assurer une surveillance accrue de la qualité des eaux en effectuant des analyses régulières de la qualité des effluents et des eaux réceptives, pour détecter les pollutions chroniques et accidentelles. Outre les analyses sur les effluents et les eaux réceptives qui peuvent être imposées dans le cadre des autorisations administratives de rejet, la législation des installations classées prévoit la possibilité d'imposer au coup par coup les évaluations que rendent nécessaires une pollution accidentelle ou une inobservation des conditions imposées aux rejets industriels. Le ministère chargé de l'environnement ne dispose pas en tout état de cause des moyens nécessaires pour financer lui-même de telles analyses.

L'Etat, s'appuyant notamment sur les agences de bassin doit par ailleurs encourager la réalisation de dispositifs d'épuration des rejets polluants tant par les collectivités locales que par les exploitants d'installations classées et veiller à leur bon fonctionnement. En matière d'assainissement des collectivités locales (effluents domestiques et effluents industriels raccordés aux réseaux), $50 \%$ seulement de la pollution arrive à une station d'épuration, et on n'élimine que $36 \%$ de cette pollution à traiter.

En définitive, les collectivités locales rejettent plus dans le milieu naturel, que les industriels pour les matières organiques. Bien qu'un certain nombre de grandes villes se soient dotées récemment d'une station d'épuration (Marseille, Nice, Grenoble, Strasbourg), un gros effort doit donc encore être fourni. La pollution industrielle de nature organique a quant à elle diminué de $33 \%$ entre 1975 et 1985.

\subsection{Exercice de la police municipale}

Dans le cadre des pouvoirs de police qui sont conférés aux maires par les articles L 131-2-6 et L 131-7 du code des communes pour assurer le maintien de l'ordre public, la sécurité et la salubrité publique, ces derniers sont en mesure, s'il y a lieu d'ordonner par voie d'arrêté, des coupures du réseau de distribution d'eau, de limiter l'arrosage, mais aussi tout prélèvement, y compris d'eau souterraine.

L'article L 131-13 du même code permet au préfet de se susbstituer au maire, en cas de carence de celui-ci, pour prendre toute mesure nécessaire en ses lieux et places.

Il appartient donc aux préfets et aux maires de mettre en œuvre les différents pouvoirs de police précités pour lutter contre la sécheresse. Il convient de souligner ici une difficulté majeure qui empêche l'administration de procé- 
der à une véritable répartition générale des eaux. Cette difficulté est de nature juridique : elle tient à l'affrontement dans le droit de l'eau des règles de droit public relatives aux pouvoirs de police de l'administration et des règles de droit privé relatives au droit d'usage accordé aux riverains des cours d'eau non domaniaux par l'article 644 du code civil, et au rôle conféré par l'article 645 du même code au juge civil.

C'est ainsi que la jurisprudence a maintes fois rappelé qu'il n'appartenait pas à l'administration de juger de l'étendue des droits conférés par le code civil aux propriétaires riverains des cours d'eau non domaniaux (CE 22 mars 1901 Pagés).

Les autorisations individuelles accordées, sauf en cas d'application de l'article 113 du code rural ou de l'article 2 de la loi du 16 octobre 1919, ne fixent donc pas pour les cours d'eau non domaniaux le débit maximal susceptible d'être prélevé, mais seulement le débit qui doit être laissé dans le cours d'eau.

La révision ou la suppression des autorisations existantes suppose par ailleurs la mise en œuvre d'une procédure comportant notamment une enquête hydraulique, qui n'est pas toujours compatible avec une situation d'urgence. Les dispositions des articles 109 du code rural et 26 du code du domaine public fluvial ne sont en outre pas applicables aux ouvrages concédés ou déclarés d'utilité publique. Il sera donc indispensable en cas de séchresse grave de recourir à des dispositions exceptionnelles.

\section{Le recours à des dispositions législatives exception- nelles en cas de sécheresse grave}

Une circulaire de 1976 rappel..it aux préfets la possibilité de faire des réquisitions d'eau en application de l'ordonnance du 6 janvier 1959 relative aux réquisitions de biens et de services.

Il convient de noter que la réquisition pouvait porter sur des eaux superficielles et souterraines (donc quelque soit le régime juridique des eaux) et que le but exclusif de la réquisition était d'assurer l'alimentation en eau potable.

Ces réquisitions ouvraient a priori droit à indemnité. En réalité, des accords amiables conduisaient généralement à des lâchures de la part des gestionnaires de grands barrages, en particulier Electricité de France.

Ces dispositions ne sont pas apparues suffisantes : c'est ainsi que l'article 45 de la loi du 22 juillet 1987 relative à l'organisation de la sécurité civile, à la préservation de la forêt contre l'incendie et à la prévention des risques majeurs a conféré aux préfets des pouvoirs de police exceptionnels en cas de sécheresse grave.

Cet article dispose que " en cas de sécheresse grave mettant en péril l'alimentation en eau potable des populations constatée par le ministre chargé de la police des eaux, des dérogations temporaires aux règles fixant les débits réservés des entreprises hydrauliques dans les bassins versants concernés peuvent être, en tant que de besoin, et après consultation de l'exploitant, ordonnés par le représentant de l'Etat dans le département, sans qu'il y ait lieu à paiement d'indemnité ».
La circulaire du 8 septembre 1988 a précisé les conditions d'application de cet article. Elles obéissent à deux principes :

- offrir au préfet du département concerné les moyens d'agir rapidement ;

- assurer une bonne coordination des mesures prises au niveau du bassin.

La mise en œuvre de ces mesures suit une procédure en « deux temps ».

\section{Procédure préalable à la constatation de l'état de séche- resse grave, par le ministre}

Les préfets tiennent informé de l'évolution de la situation de sécheresse, le préfet coordonnateur de bassin, dès que les circonstances laissent présumer de la nécessité de recourir aux dispositions de l'article 45 de la loi du 22 juillet 1987.

Si les circonstances le nécessitent dans son département, le préfet concerné doit saisir le ministre chargé de la police des eaux en application de l'article 45 de la loi du 12 juillet 1987. Cette demande doit être accompagnée d'un rapport justificatif : ce rapport doit témoigner de ce que toutes les autres mesures possibles ont été préalablement mises en œuvre, car l'application de l'article 45 de la loi du 12 juillet 1987 doit rester exceptionnelle. Copie de ce rapport est adressée au préfet coordonnateur de bassin pour qu'il fasse part de son avis au secrétaire d'Etat.

La décision de constatation de l'état de sécheresse grave est prise par le ministre chargé de la police des eaux et notifiée au préfet concerné dans les meilleurs délais.

\section{Mesures susceptibles d'être prises par le préfet en appli- cation de l'article 45 de la loi du 22 juillet 1987}

L'état de sécheresse grave ayant été constaté, il appartient au préfet de prescrire les dérogations temporaires aux règles fixant les débits réservés qu'il estime nécessaire.

Les mesures prescrites aux exploitants concernés, qui ne peuvent avoir pour objectif que de satisfaire aux besoins d'alimentation en eau destinée à la consommation humaine, peuvent consister soit en un relèvement du débit réservé sortant d'un barrage-réservoir, soit en un changement apporté aux modulations de ce débit réservé. Il peut s'agir également de la possibilité ouverte à la collectivité ou à l'établissement, maître d'une prise d'alimentation en eau destinée à la consommation humaine, d'abaisser le débit à maintenir à l'aval de cette prise en dessous des débits réservés fixés par l'autorisation, la concession ou les dispositions de l'article 410.

En application de l'article 45 de la loi du 22 juillet 1987 , ces mesures ne peuvent donner lieu à indemnité.

On voit que la loi donne aux préfets un large pouvoir d'appréciation quant aux mesures à mettre en œuvre : il appartient donc à celui-ci de faire un examen préalable attentif de l'ensemble des utilisations de l'eau et des milieux aquatiques sur le tronçon du cours d'eau concerné, et d'en assurer la cohérence, en particulier lorsque le cours d'eau traverse plusieurs départements. 


\begin{tabular}{|c|c|c|c|}
\hline \multicolumn{2}{|c|}{ Le réseau hydrographique en métropole } \\
\hline Nature des cours d'eau & $\begin{array}{c}\text { Inscrits à la nomenclature des } \\
\text { cours d'eau navigables }\end{array}$ & $\begin{array}{c}\text { Rayés de la nomenclature des } \\
\text { cours d'eau navigables }\end{array}$ & $\begin{array}{c}\text { TOTAUX } \\
\mathrm{km}\end{array}$ \\
\hline \multirow{2}{*}{ DOMANIAUX } & $\begin{array}{c}\text { canaux }-4500 \mathrm{~km} \\
\text { (dont } 283 \mathrm{~km} \text { concédés) } \\
\text { rivières }-5500 \mathrm{~km} \\
\text { (dont } 373 \mathrm{~km} \text { concédés) }\end{array}$ & $\begin{array}{c}\text { canaux }-450 \mathrm{~km} \\
\text { (dont } 190 \mathrm{~km} \text { concédés) } \\
\text { rivières }-6500 \mathrm{~km} \\
\text { (dont } 595 \mathrm{~km} \text { concédés) }\end{array}$ & 4950 \\
\cline { 2 - 4 } & \multicolumn{1}{|c|}{$6000 \mathrm{~km}$} & D'une largeur supérieure à $1,00 \mathrm{~m}: 110000 \mathrm{~km}$ & 16950 \\
\hline NON & D'une largeur supérieure à $0,50 \mathrm{~m}: 150000 \mathrm{~km}$ & 260000 \\
\hline
\end{tabular}

L'arrêté (ou les arrêtés) pris en application de l'article 45 sera notifié sans délai aux propriétaires ou aux exploitants des entreprises, ainsi qu'aux représentants des collectivités locales et des établissements ayant pour vocation l'alimentation en eau destinée à la consommation humaine.

Cet arrêté ainsi que l'arrêté constatant l'état de sécheresse grave, devront être rapportés dès que les circonstances qui justifiaient les dérogations ordonnées ne seront plus réunies.

\section{Conclusion}

Le droit français de l'eau a bien, dans le cas d'une sécheresse grave, privilégié l'alimentation en eau potable, mais il convient de conserver aux dispositions de l'article 45 un caractère exceptionnel : les préfets doivent donc utiliser les pouvoirs de police qui leur incombent pour gérer au mieux la ressource et éviter les gaspillages.

En réalité, on ne peut régler véritablement les problèmes de répartition générale des eaux avec les dispositions législatives existantes. Ces problèmes de répartition des eaux ne peuvent être réglés que dans le cadre de démarches contractuelles, du type du contrat-Charente. On ne saurait trop insister sur l'intérêt que présente par ailleurs une politique de gestion globale de la rivière.

Le droit français de l'eau ne facilite pas la gestion de la sécheresse. Il convient d'examiner avec attention les dispositions juridiques à mettre en œuvre pour permettre à l'Etat d'assurer une gestion efficace des eaux. La réflexion en cours sur la modernisation du droit de l'eau s'inscrit dans cette perspective. Il pourrait par exemple être proposé que les règlements d'eau comportent la fixation du débit maximum prélevé.

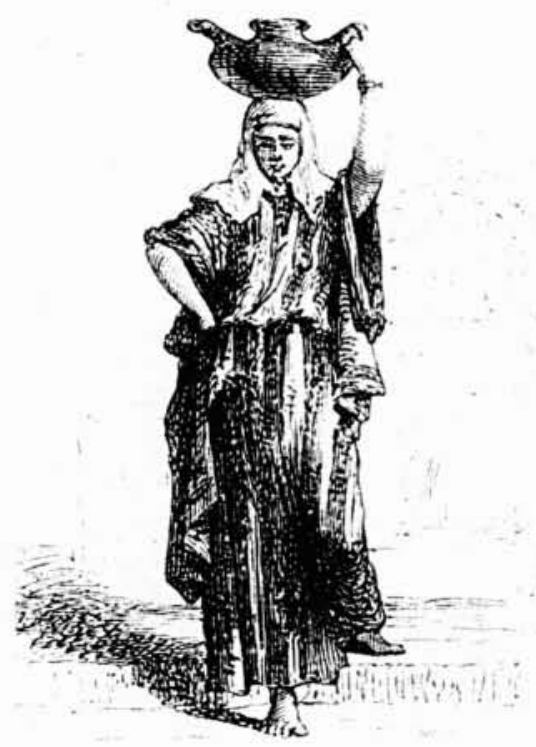

